\title{
Interdiffusion of Solvent into Glassy Polymer Films: A Molecular Dynamics Study
}

\author{
Mesfin Tsige* and Gary S. Grest ${ }^{\dagger}$ \\ Sandia National Laboratories, Albuquerque, NM 87185
}

(Dated: October 29, 2018)

\begin{abstract}
Large scale molecular dynamics and grand canonical Monte Carlo simulation techniques are used to study the behavior of the interdiffusion of a solvent into an entangled polymer matrix as the state of the polymer changes from a melt to a glass. The weight gain by the polymer increases with time $t$ as $t^{1 / 2}$ in agreement with Fickian diffusion for all cases studied, although the diffusivity is found to be strongly concentration dependent especially as one approaches the glass transition temperature of the polymer. The diffusivity as a function of solvent concentration determined using the one-dimensional Fick's model of the diffusion equation is compared to the diffusivity calculated using the Darken equation from simulations of equilibrated solvent-polymer solutions. The diffusivity calculated using these two different approaches are in good agreement. The behavior of the diffusivity strongly depends on the state of the polymer and is related to the shape of the solvent concentration profile.
\end{abstract}

\footnotetext{
* mtsige@sandia.gov

† gsgrest@sandia.gov
} 


\section{INTRODUCTION}

Understanding the interdiffusion of solvent into a polymer is crucial for a variety of applications, such as food storage, controlled drug release and membrane separations. ${ }^{1.2 .3 .4 .5}$ The mechanisms controlling the interdiffusion process are reasonably well understood ${ }^{6.7 .8 .9}$ but predicting accurately the nature of the diffusion has been a challenging problem. It is now widely accepted that the interdiffusion of solvent into a polymer depends on solvent concentration gradient within the system as well as the rate of polymer segmental relaxation. ${ }^{10.11 .12 .13 .14}$ Whether the polymer is a melt above its glass transition temperature $T_{g}$ or an amorphous solid below $T_{g}$ strongly affects the diffusion behavior 10

In general, three categories of diffusion behavior of solvents into polymers have been distinguished. $\frac{9,15,16}{}$ These are, Fickian or Case I, Case II or Class II, and anomalous diffusion: in which the rate of diffusion of solvent is much less than, much greater than, or comparable to the rate of polymer segmental relaxation, respectively. A simple descriptive way of quantifying these is based on the power law dependence of the mass uptake of the solvent by the polymer or the distance covered by the solvent as a function of time $t\left(\sim t^{n}\right)$. For Fickian diffusion $n=1 / 2$, for Class II diffusion $n=1$, and for anomalous diffusion $1 / 2<n<1$. Fickian diffusion usually applies for all solvent concentration when the solvent interdiffuses into a polymer melt, while for glassy polymers it usually applies only for low solvent concentration. Non-Fickian kinetics is expected when the viscoelastic properties of the system becomes the determining factor ${ }^{10.11}$ In addition to linear kinetics, Case II diffusion is characterized by a sharp concentration front that propagates at constant speed 11.17 .18 with a Fickian type precursor foot ${ }^{8.15 .18 .19}$ preceding the front.

When a solvent film is placed in contact with one surface of a polymer melt, the diffusion is one-dimensional and can often be described by Fick's one-dimensional diffusion equation ${ }^{20}$

$$
\frac{\partial c}{\partial t}=\frac{\partial}{\partial z}\left(D(c) \frac{\partial c}{\partial z}\right)
$$

where $c$ is the solvent concentration in units of mass per unit volume and $D(c)$ is the diffusivity. This equation assumes that the volume of the medium is not changed by the interdiffusion of the solvent. If $D(c)$ is a function of $c$ only, then the Boltzmann transformation of Eq. 1] gives

$$
\frac{\partial z}{\partial t}=f(D, c) t^{1 / 2}
$$


where $f(D, c)$ is a function of $D$ and $c$ only. This equation reflects the square root time dependence of Fickian diffusion irrespective of the functional form of $D(c)$. It can be integrated to yield the diffusion coefficient at concentration $c^{\prime 20}$

$$
D\left(c^{\prime}\right)=-\frac{1}{2}\left[\left(\frac{d c}{d \eta}\right)\right]_{c^{\prime}}^{-1} \int_{0}^{c^{\prime}} \eta d c
$$

where $\eta=z / t^{1 / 2}$. Thus from the scaled concentration profile one can directly obtain the diffusivity $D(c)$. Note that only in a few special cases, like $D(c)$ constant, can Eq. 1 be solved analytically ${ }^{20}$ For the case in which $D(c)$ is a constant, $c(\eta)$ is an error function. However, it is important to remember that Fickian diffusion, i.e. uptake increasing as $t^{1 / 2}$, is true provided that the diffusivity depends only on $c$ as shown above.

The diffusivity can also be approximately obtained from the following Darken equation applied to solvent diffusing in an equilibrated polymer solution

$$
D(c)=D_{c}(c)\left(\frac{\partial \ln f}{\partial \ln c}\right)_{T}
$$

where $D_{c}(c)$ is the corrected diffusion constant and $f$ is the fugacity of the solvent, both are defined in the next section.

Molecular dynamics (MD) simulation technique is proven to be a useful tool for determining the diffusion coefficients of penetrant molecules in polymers. This technique is specially important when detailed microscopic information of the mechanism of transport is required. Most of the previous studies have focused on the penetrant transport of small molecules in a polymer melt. $3,5,21,22,23,24,25,26,27,28,29,30,31$ With recent advances in parallel molecular dynamics algorithms and the increased computational power, progress has primarily occurred for studying the diffusion of large molecules (phenol molecules) in a polymeric matrix at atomistic level. ${ }^{31,32}$ However, equivalent development is lacking for interdiffusion of solvent into polymer or polymer-polymer interdiffusion. In the previous study, which will be referred to hereafter as paper $\mathrm{I},{ }^{33}$ we investigated the interdiffusion of a solvent into a homopolymer melt. The solvent concentration profile and weight gain by the polymer was measured as a function of time. The weight gain was found to scale as $t^{1 / 2}$ and the concentration profiles were found to fit very well assuming Ficks's second law with constant diffusivity. The study, however, focused only on homopolymers that are far above the glass transition temperature.

In this paper we extend our previous study on interdiffusion of solvent into homopolymers that are close to the glass transition temperature of the homopolymer. Case II diffusion has 
been inaccessible to computer simulation due to the extensive computational effort required. In this paper we study the conditions that may lead to Case II type diffusion behavior. The main purpose of the present study is two-fold: first, to understand how the dependence of the diffusivity $D(c)$ on concentration changes as the state of the polymer changes and also its relation to the form of the concentration profile curve; second, to test the Darken approach under general conditions where $D_{c}(c)$ is not a constant. As in paper I, we are also interested in the relationship between the self-diffusion constant of the solvent and the corrected diffusion and the diffusivity.

The outline of this paper is as follows. In Sec. II a brief review of the molecular dynamics simulation and the model used is given. In Sec. III the interdiffusion results for different polymer-polymer and solvent-polymer interaction parameters are presented and discussed. The diffusivity $D(c)$ calculated from solvent concentration profiles and from the Darken equation are presented in Sec. IV. The self- and corrected diffusion constants as a function of solvent concentration are also presented and discussed. The main results of the present study are summarized in Sec. V.

\section{SIMULATION DETAILS}

\section{A. System}

The basic model of the polymer-solvent system is the same as used in paper I. The polymer is treated as freely jointed bead-spring chain of length $N$ monomers of mass $m$ and the solvent is modeled as single monomer of mass $m$. All monomers of type $\alpha$ and $\beta$ interacts through the standard Lennard-Jones 6-12 potential

$$
U_{L J}(r)= \begin{cases}4 \epsilon_{\alpha \beta}\left\{\left(\frac{\sigma_{\alpha \beta}}{r}\right)^{12}-\left(\frac{\sigma_{\alpha \beta}}{r}\right)^{6}\right\}+\epsilon_{L J}, & r \leq r_{c} \\ 0, & r>r_{c}\end{cases}
$$

where $r$ is the distance between monomers and $\epsilon_{L J}$ is a constant added so that the potential is continuous at $r=r_{c}$. Here $\alpha=p$ stands for the polymer monomer and $\alpha=s$ for a solvent monomer. $\epsilon_{s s}=\epsilon$ and $\sigma$ define the units of energy and length, respectively. Here we take $\sigma=\sigma_{\alpha \beta}$ and $r_{c}=2.5 \sigma$. For our model, the freezing temperature of the solvent is higher than the glass transition temperature of a long fully flexible polymer melt, $T_{g}=0.5-0.6 \epsilon / k_{B} \cdot \underline{\underline{34}}$ Thus, temperature is not a good variable to change the state of the polymer without changing 
the state of the solvent. Instead, we vary $\epsilon_{p p}$ from $\epsilon_{p p}=\epsilon$ (melt) to $2 \epsilon$ (glassy). Berthelot rule $\epsilon_{s p}=\sqrt{\epsilon_{s s} \epsilon_{p p}}$ is used for the cross term in some cases but we also study other cross terms. This is because $\left(\epsilon_{p p}, \epsilon_{s p}\right)=(2 \epsilon, \sqrt{2} \epsilon)$ is found to be immiscible except in the dilute limit. As in paper I, for bonded monomers an additional anharmonic potential known as FENE potential with $R_{0}=1.5 \sigma$ and $k=30 \epsilon$ is applied. $\underline{35.36}$

For the interdiffusion of solvent monomers into a polymeric matrix, the system consist of entangled polymer chains in a rectangular box which is periodic in $x$ and $y$ but not in $z$, the diffusion direction. This initial configuration was generated following the procedure given in paper I. The polymer consisted of 600 chains of length $N=500$ monomers. The solvent consisted of 230,000 monomers. For the self- and corrected diffusion studies as a function of concentration, the system consist of an equilibrated polymer solvent mixture in a cubic box which is periodic in all directions. The polymer in the system consisted of $M$ chains of length $N=500$ monomers, where $M=100$ for solvent concentration $c<0.45 \sigma^{-3}$ and $M=50$ for $c \geq 0.45 \sigma^{-3}$. The mole fraction of solvent $x_{s}$ in the mixture was varied from 0.01 (dilute case) to 0.75 . A pure solvent system of 50,000 monomers was also simulated.

In paper I we compared results from Langevin thermostat simulations which screens the hydrodynamic interactions with results from dissipative particle dynamics (DPD) thermostat simulations, which does not. The results from the two thermostats agreed when the dissipation from the thermostats become much smaller than from particle collisions. In the present study, to conserve hydrodynamic interactions, we use DPD thermostat through out the simulation. For details see paper I. The equations of motion were integrated with a velocity verlet algorithm with a time step of $\Delta t=0.012 \tau$ for the interdiffusion study and $\Delta t=0.009 \tau$ for the bulk equilibrium measurement of the self- and corrected diffusion constants, where $\tau=m(\sigma / \epsilon)^{1 / 2}$. All the simulations were run using the massively parallel code LAMMPS 37 at a temperature of $T=\epsilon / k_{B}$ and pressure $P \simeq 0$ without tail corrections to be comparable to interdiffusion - same as paper I.

\section{B. Diffusion Coefficients}

We have calculated the self-and corrected diffusion coefficients and diffusivity $D(c)$ of solvents in an equilibrated solvent polymer mixture as a function of solvent concentration, $c$. The self-diffusion constant $D_{s}(c)$ of the solvent in the polymer was calculated from the 
slope of the solvent mean square displacement

$$
D_{s}(c)=\lim _{t \rightarrow \infty} \frac{\left\langle[\boldsymbol{r}(t)-\boldsymbol{r}(0)]^{2}\right\rangle}{6 t} .
$$

Here $\langle\ldots\rangle$ denote an ensemble average and is obtained by averaging over all solvents and many initial time origins, and $\boldsymbol{r}(t)$ is the position of a solvent in the polymer at time $t$.

The corrected diffusion coefficient $D_{c}(c)$ of the solvent in the polymer is calculated using the Einstein form equation ${ }^{33}$

$$
\begin{aligned}
D_{c}(c)= & N_{T} x_{s} x_{p} \lim _{t \rightarrow \infty} \frac{1}{6 t}\left\langle\left\{\left[\boldsymbol{r}_{c m, s}(t)-\boldsymbol{r}_{c m, s}(0)\right]\right.\right. \\
& \left.\left.-\left[\boldsymbol{r}_{c m, p}(t)-\boldsymbol{r}_{c m, p}(0)\right]\right\}^{2}\right\rangle
\end{aligned}
$$

where $x_{i}$ and $\boldsymbol{r}_{c m, i}(t)$ are mole fraction and center of mass of all monomers of species $i$ at time $t$, respectively, and $N_{T}=N_{s}+N_{p}$ is the total number of monomers.

To determine the fugacity $f$ the particle insertion method ${ }^{38}$ is applied using the grand canonical MD code LADERA. ${ }^{39}$ During the course of an equilibrium molecular dynamics simulation at the appropriate solvent concentration, the energy, $E$, of inserting a solvent particle at random locations was sampled. The excess chemical potential energy $\mu_{e}$ is computed using

$$
\mu_{e}=-k_{B} T \ln \left\langle\exp \left(-E / k_{B} T\right)\right\rangle,
$$

where $k_{B}$ is the Boltzmann constant, $T$ is the temperature and $\langle\ldots\rangle$ is an ensemble average. Then, the activity coefficient, $\gamma$, is computed via $\gamma=\exp \left(\mu_{e} / k_{B} T\right)$. The thermodynamic factor in eq. 4 can be expressed in terms of the activity coefficient $\gamma$ of the solvent as

$$
\frac{\partial \ln f}{\partial \ln c}=1+\frac{\partial \ln \gamma}{\partial \ln c}
$$

The thermodynamic factor goes to 1 as $c \rightarrow 0$.

The computing time depends on the state of the polymer where much longer run are required as the effective temperature of the polymer melt is reduced towards its glass transition temperature. At the lowest temperature studied and for a given solvent concentration $c$ a run of about half a million MD time steps are required to calculate the self-diffusion constant $D_{s}(c)$ while a run of more than four million MD time steps is required for the corrected diffusion constant $D_{c}(c)$. The fugacity calculation at each solvent concentration requires more than four million $\mathrm{MC}$ insertion attempts. For the interdiffusion simulations, 
one run was made for each set of parameters specified. Each system is run until the solvent reaches the lower substrate and on the average requires a run of about five million MD time steps.

\section{INTERDIFFUSION}

Interdiffusion studies of a solvent into an equilibrated polymer has been conducted for different cases of polymer-polymer and solvent-polymer interactions. The initial setup for the interdiffusion study is the same as Fig. 1 of paper I. The density profile of both polymer and solvent as a function of time for $k_{B} T / \epsilon_{p p}=1.0,0.75$ and 0.5 with Berthelot's rule for the cross-term $\epsilon_{s p}$ is shown in Fig. 1. The solvent diffuses into the polymer from the right side. As the state of the polymer changes from melt $\left(k_{B} T / \epsilon_{p p}=1.0\right)$ to glass $\left(k_{B} T / \epsilon_{p p}=0.5\right)$ the solvent density profile changes to a sharp front. The solvent diffusion is Fickian in all the three cases as confirmed by the linearity of the weight gain by the polymer versus $t^{1 / 2}$ curve shown in Fig. 2. This indicates that the precursor of the front for $k_{B} T / \epsilon_{p p}=0.5$ is Fickian. This is in agreement with recent experimental observations that characterize Case II diffusion by a sharp concentration front with a Fickian type precursor ${ }^{8.15 .18 .19}$ For the front to move the solvent mobility should be much greater than the rate of polymer segmental relaxation. ${ }^{10}$ But, for this case the front does not move in the time scale of our simulation. In fact, simulation of an equilibrated solvent-polymer solution, discussed in the next section, shows that only a small amount of solvent is soluble for this case suggesting that the front may not move at all.

In order to facilitate the interdiffusion of solvent into the glassy polymer $\left(k_{B} T / \epsilon_{p p}=0.5\right)$, the interaction between polymer and solvent is increased to $\epsilon_{s p}=1.55 \epsilon, 1.7 \epsilon$, and $2.0 \epsilon$. Similarly $\epsilon_{s p}$ is increased to $1.33 \epsilon$ for $k_{B} T / \epsilon_{p p}=0.75$. The corresponding density profiles of both polymer and solvent as a function of time is shown in Fig. 3. We clearly see that as $\epsilon_{s p}$ is increased the solubility is enhanced for both $\epsilon_{p p}=1.33 \epsilon$ and $2.0 \epsilon$ and the density front observed for $\left(\epsilon_{p p}, \epsilon_{s p}\right)=(2.0 \epsilon, \sqrt{2.0} \epsilon)$ disappears. The $\epsilon_{s p}=1.7 \epsilon$ case shows a cross-over. There is no change in the diffusion process due to the change in $\epsilon_{s p}$ as the weight gain by the polymer system for all four cases increases as $t^{1 / 2}$, see Fig. 2, in agreement with Fickian diffusion. 


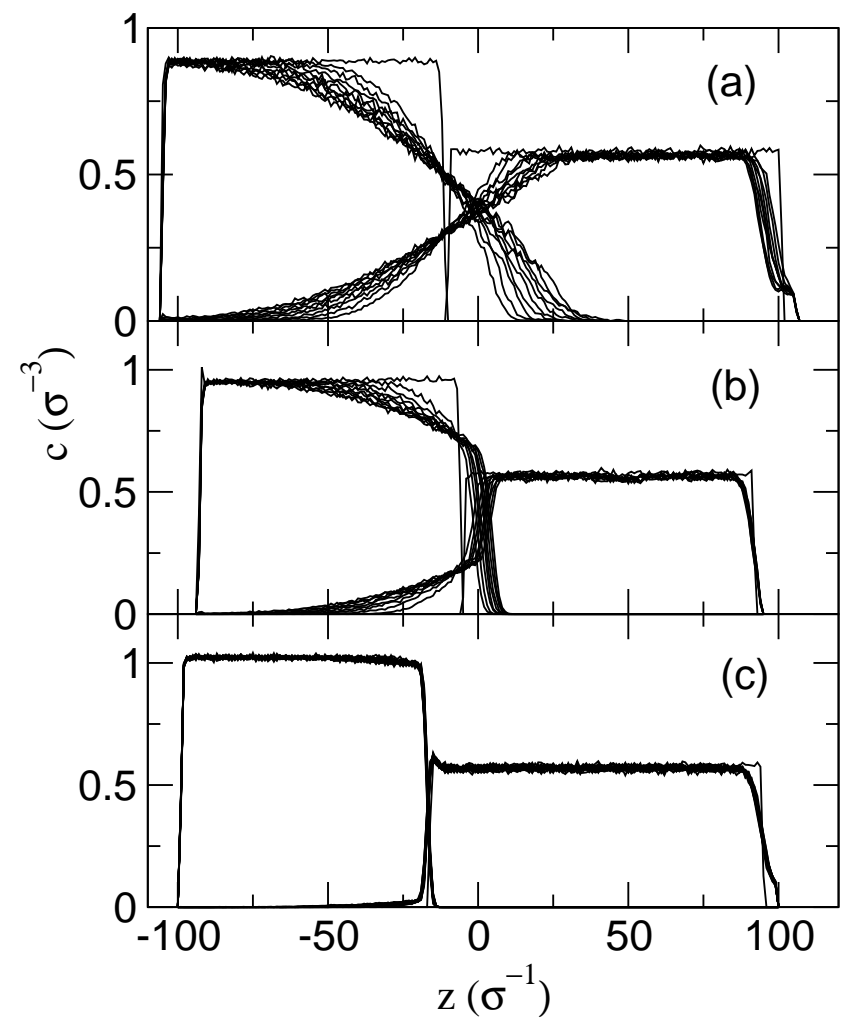

FIG. 1: Solvent and polymer concentration profiles as a function of time for (a) $k_{B} T / \epsilon_{p p}=1.0$, plotted every $2400 \tau$, (b) $k_{B} T / \epsilon_{p p}=0.75$, plotted every $4800 \tau$, and (c) $k_{B} T / \epsilon_{p p}=0.5$, plotted every $12000 \tau$, with Berthelot's rule $\epsilon_{s p}=\sqrt{\epsilon_{p p} \epsilon_{s s}}$ for the cross-term. The solvent diffusing into the polymer from the right side.

\section{DIFFUSION COEFFICIENTS}

The diffusivity of the solvent can be calculated from the solvent concentration profile using Eq. 3. It can be also calculated from simulation of equilibrated solvent-polymer solution using the Darken equation (Eq. 4). In this section we compare diffusivity results from the two different approaches.

\section{A. Diffusivity from concentration profile}

Using the change of variable $\eta=z t^{-1 / 2}$ the solvent density profiles corresponding to different times superimpose as shown in Fig. 4. This indicates that the solvent diffusivity is independent of position as expected for Fickian diffusion. It also means that the solvent 


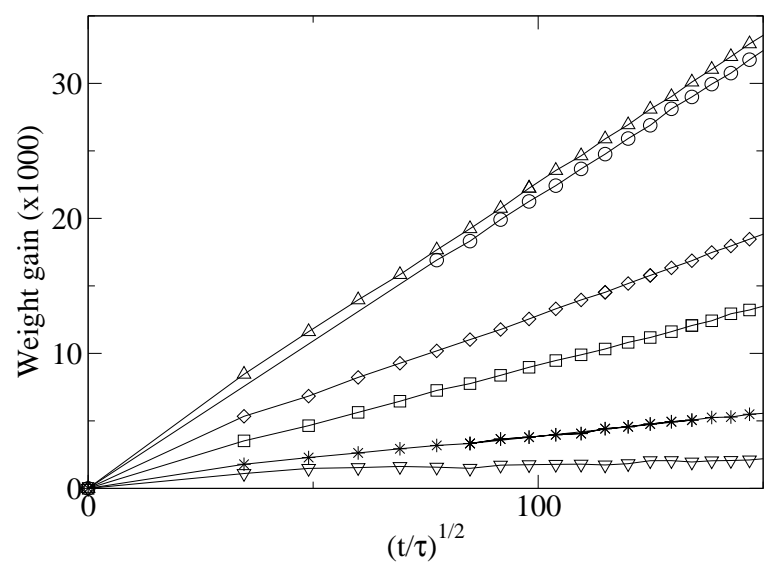

FIG. 2: Weight gain in terms of the number of solvent monomers diffused into the polymer at a given time $t$, for $\left(\epsilon_{p p}, \epsilon_{s p}\right)=(\epsilon, \epsilon)$ (circles), $(1.33 \epsilon, \sqrt{1.33} \epsilon)$ (squares), (2.0 $\left.\epsilon, \sqrt{2.0} \epsilon\right)$ (down triangles), $(2.0 \epsilon, 1.55 \epsilon)$ (stars), $(2.0 \epsilon, 1.7 \epsilon)$ (diamond), and $(2.0 \epsilon, 2.0 \epsilon)$ (up triangles), $T=\epsilon / k_{B}$.

diffusivity for absorption can be determined as a function of concentration by integrating Eq. 3 with respect to $\eta$.

To calculate $D(c)$ analytically using Eq. 3, the average of the transformed solvent density profiles $c(\eta)$ of each case shown in Fig. 4 was fit to a polynomial function of at least order 5 . This higher order polynomial was chosen to get an optimal fit to the concentration profiles. The data is integrated analytically up to the target concentration using the transformation $\int_{0}^{c^{\prime}} \eta d c=\int_{\eta_{0}}^{\eta} \eta \frac{d c}{d \eta} d \eta$. This procedure is repeated for different values of solvent concentration and the diffusivity $D(c)$ calculated for the different cases is shown in Fig. 5 ,

In general, the behavior of $D(c)$ strongly depends on the state of the polymer. The diffusivity is approximately a constant when the homopolymer is far above the glass transition, $T_{g}$, that is for $k_{B} T / \epsilon_{p p}=1.0$, and then becomes concentration dependent as the effective temperature of the polymer melt $k_{B} T / \epsilon_{p p}$ is reduced towards its glass transition temperature. For $\left(\epsilon_{p p}, \epsilon_{s p}\right)=(1.33 \epsilon, \sqrt{1.33} \epsilon),(2.0 \epsilon, \sqrt{2.0} \epsilon)$ and $(2.0 \epsilon, 1.7 \epsilon)$ the calculated diffusivity for concentrations in the steep part of the concentration profile (not included in Fig. 5 ) resulted in large scatter of the data. This is because little variation of the slope in this region introduces large error in the diffusivity.

For $\epsilon_{p p}=1.33 \epsilon$, the diffusivity is approximately a constant for $\epsilon_{s p}=\sqrt{1.33} \epsilon$, but increases linearly with concentration for $\epsilon_{s p}=1.33 \epsilon$. Note that in the dilute limit $(c \rightarrow 0)$ the diffusivity is independent of $\epsilon_{s p}$. For $\epsilon_{p p}=2.0 \epsilon$, the diffusivity is independent of $\epsilon_{s p}$ within the 


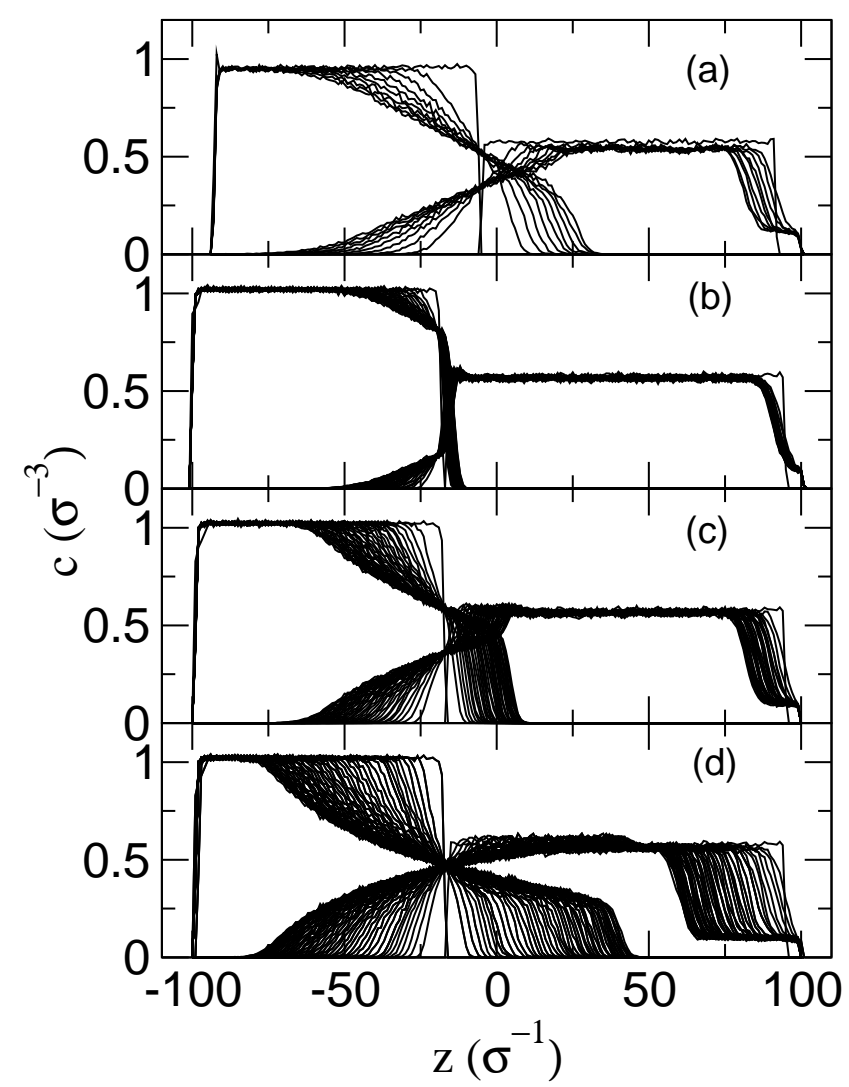

FIG. 3: Solvent $\rho_{s}$ and polymer $\rho_{p}$ concentration profiles as a function of time, plotted every $2400 \tau$ for $\left(\epsilon_{p p}, \epsilon_{s p}\right)=(\mathrm{a})(1.33 \epsilon, 1.33 \epsilon)$, (b) $(2.0 \epsilon, 1.55 \epsilon)$, (c) $(2.0 \epsilon, 1.7 \epsilon)$, and (d) $(2.0 \epsilon, 2.0 \epsilon)$.

error of the simulation. For this case the diffusivity can be approximated by an exponential function of the form $D(c)=D_{0} \exp (\alpha c)$, where $D_{0}$ and $\alpha$ are constants that may depend on

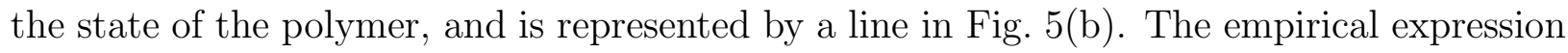
found from fitting the diffusivity curve of a given system was in turn used to solve Eq. 1

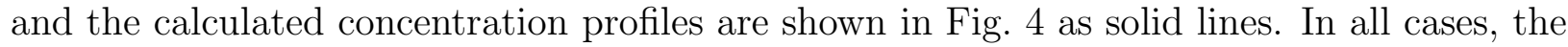
calculated concentration profiles give an adequate description of the simulated concentration profiles in the region of interest.

The behavior of $D(c)$ is directly related to the form of the concentration profile curve. When the solvent concentration profile is concave (i.e. for $\left(\epsilon_{p p}, \epsilon_{s p}\right)=(\epsilon, \epsilon)$ and $(1.33 \epsilon, \sqrt{1.33} \epsilon))$ the diffusivity is approximately a constant. However, when the diffusivity shows exponential dependence on solvent concentration (for $\epsilon_{p p}=2.0 \epsilon$ ) then the solvent concentration profile curve becomes convex. For the case in which the concentration is approximately linear $\left(\epsilon_{p p}=\epsilon_{s p}=1.33 \epsilon\right)$, the diffusivity increases linearly with concentration. 


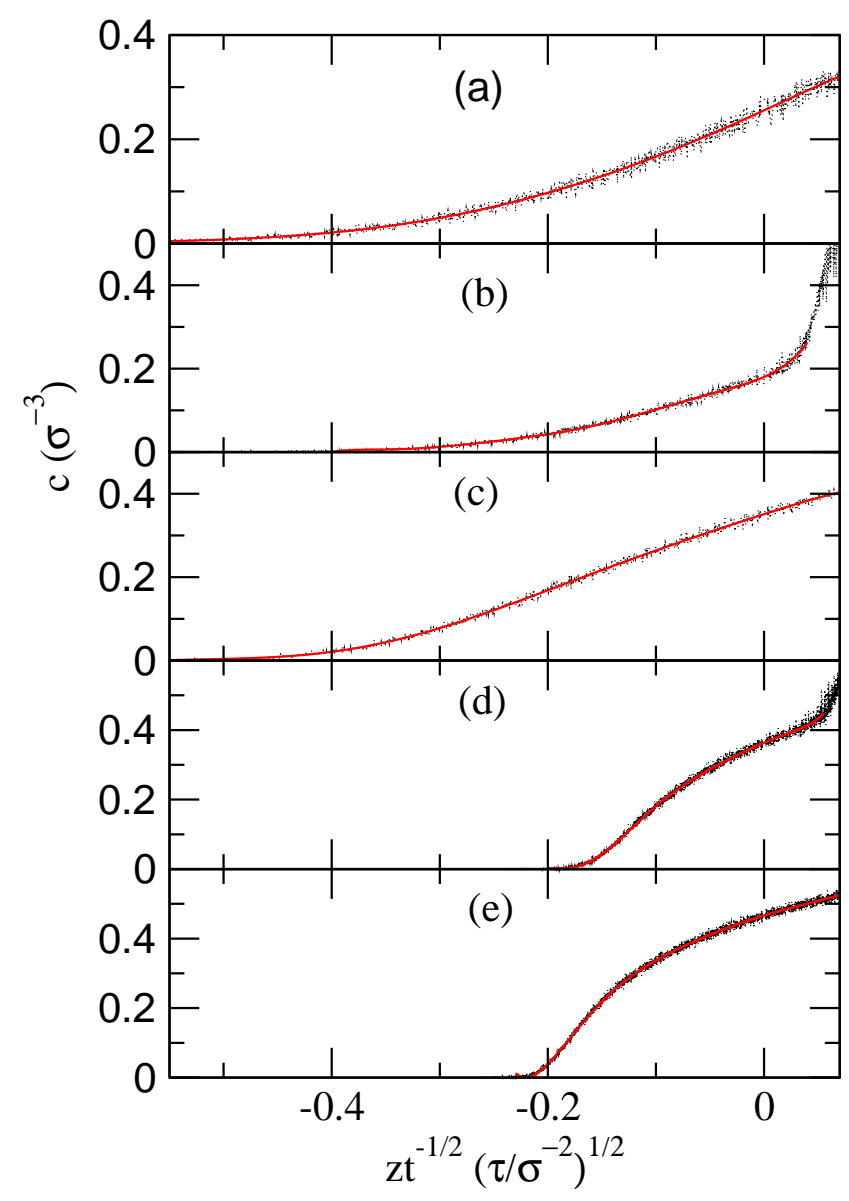

FIG. 4: [Color online] Solvent concentration profiles are plotted as a function of the scaling variable $z t^{-1 / 2}$ for $\left(\epsilon_{p p}, \epsilon_{s p}\right)=(\mathrm{a})(\epsilon, \epsilon)$, (b) $(1.33 \epsilon, \sqrt{1.33} \epsilon),(\mathrm{c})(1.33 \epsilon, 1.33 \epsilon)$, (d) $(2.0 \epsilon, 1.7 \epsilon)$, and (e) $(2.0 \epsilon, 2.0 \epsilon)$. The red (light dark) solid lines represent the theoretical curve based on the solution of Eq. 3

Numerical solution of Eq. 1 for a given functional form of diffusivity on concentration has shown a similar relation between the shape of the concentration profile and the dependence of diffusivity on concentration. ${ }^{20}$

\section{B. Diffusion Coefficients from Equilibrated Polymer solution simulations}

Self- and corrected diffusion coefficients. The self-diffusion, $D_{s}(c)$, and corrected diffusion, $D_{c}(c)$, coefficients are calculated from Eq. 6] and Eq. 7. respectively. $D_{s}(c)$ and $D_{c}(c)$ as a function of solvent concentration for different polymer-polymer and solvent- 


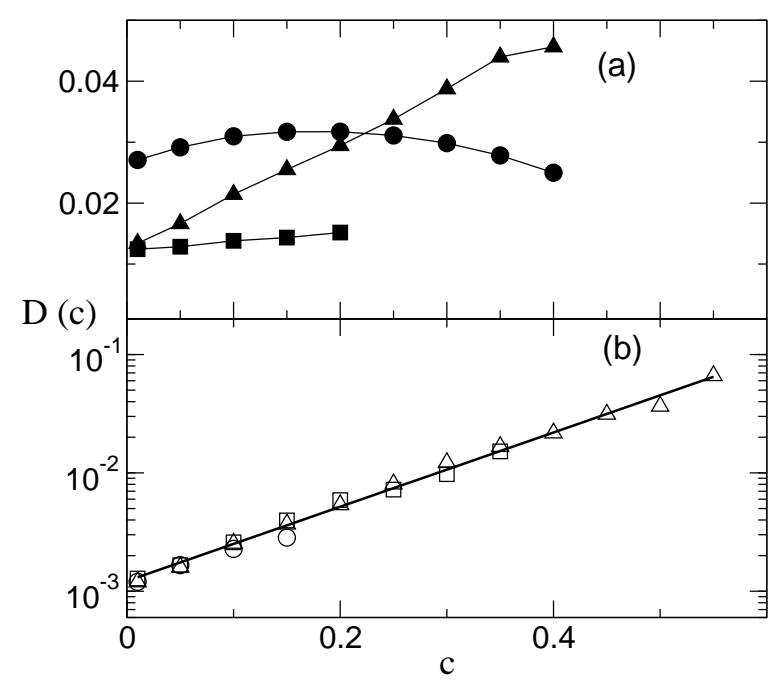

FIG. 5: Diffusivity $D(c)$ as a function of solvent concentration calculated from the solvent concentration profile using Eq. 3. The different symbols are for $\left(\epsilon_{p p}, \epsilon_{s p}\right)=(\epsilon, \epsilon)$ (closed circles), (1.33 $\epsilon$, $\sqrt{1.33} \epsilon)$ (closed squares), (1.33 $\epsilon, 1.33 \epsilon)$ (closed triangles), $(2.0 \epsilon, 1.55 \epsilon)$ (open circles), $(2.0 \epsilon, 1.7 \epsilon)$ (open squares), and $(2.0 \epsilon, 2.0 \epsilon)$ (open triangles). The solid line in (b) is an exponential fit to the data.

polymer interactions are shown in Fig. 6(a) and (b), respectively. Note that the diffusion coefficients calculated for $\left(\epsilon_{p p}, \epsilon_{s p}\right)=(1.33 \epsilon, \sqrt{1.33} \epsilon)$ and $(2.0 \epsilon, \sqrt{2.0} \epsilon)$ is limited to low solvent concentration since the systems phase separate for large $c$. The critical value of solvent concentration above which the system phase separates can be approximately determined directly from the behavior of the solvent concentration profile shown in Fig. 11 and 3. We have observed that for $\left(\epsilon_{p p}, \epsilon_{s p}\right)=(1.33 \epsilon, \sqrt{1.33} \epsilon),(2.0 \epsilon, \sqrt{2.0} \epsilon)$, and $(2.0 \epsilon, 1.7 \epsilon)$ the system phase separates for concentration values corresponding to the steep part of the concentration profile. The critical solvent concentration value is approximately the concentration value corresponding to the inflection point of the concentration curve. The critical solvent concentration value for $\left(\epsilon_{p p}, \epsilon_{s p}\right)=(2.0 \epsilon, \sqrt{2.0} \epsilon)$ is basically the dilute limit.

In general, the self and corrected diffusion coefficients shown in Fig. 6] show an exponential dependence on concentration. However, for $\left(\epsilon_{p p}, \epsilon_{s p}\right)=(\epsilon, \epsilon)$ and $(1.33 \epsilon, \sqrt{1.33} \epsilon)$ the dependence of the corrected diffusion on concentration is weak at low solvent concentration. For a given value of concentration, as expected, both $D_{s}(c)$ and $D_{c}(c)$ decrease as the state of the polymer changes from melt to glassy. Note that $D_{s}(0) \simeq D_{c}(0)$ in all cases. 


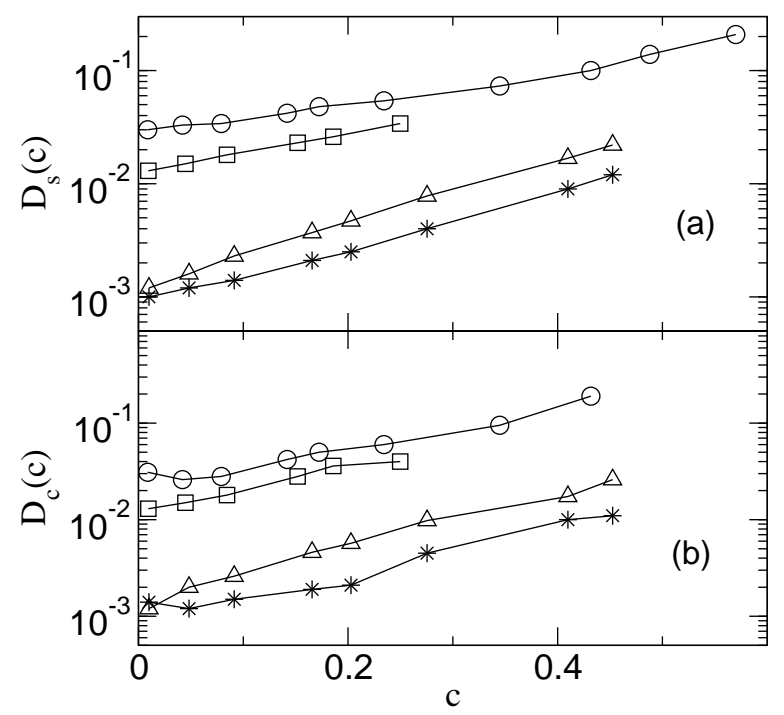

FIG. 6: Dependence of diffusion constants on solvent concentration, (a) $D_{s}(c)$ and (b) $D_{c}(c)$. Symbols are for $\left(\epsilon_{p p}, \epsilon_{s p}\right)=(\epsilon, \epsilon)$ (circles), $(1.33 \epsilon, \sqrt{1.33} \epsilon)$ (squares), $(2.0 \epsilon, 1.7 \epsilon)$ (triangles), and $(2.0 \epsilon, 2.0 \epsilon)$ (stars).

Diffusivity. To calculate the diffusivity $D(c)$ using Eq. 4, the thermodynamic factor given by Eq. 9 has to be first determined. Using the GCMD simulation method, the activity coefficient of the solvent is determined as a function of solvent concentration and is shown in Fig. 7 on a ln-ln plot. Note that the concentration at which all the activity coefficients converge is the pure solvent case. As expected, the activity coefficient is constant for low solvent concentration and thus $D(0) \approx D_{c}(0)=D_{s}(0)$ for all cases. As the solvent concentration increases the activity coefficient for $\left(\epsilon_{p p}, \epsilon_{s p}\right)=(\epsilon, \epsilon)$ and $(1.33 \epsilon, \sqrt{1.33} \epsilon)$ decreases with solvent concentration and increases for $\epsilon_{p p}=\epsilon_{s p}=1.33 \epsilon$ and $2.0 \epsilon$. This results in the diffusivity for the former two cases to be lower while for the latter two cases to be higher than the corrected diffusion constant. However, the curves in Fig. [7 are not smooth, making it difficult to determine the thermodynamic factor and thus the diffusivity with high precession.

Instead of numerically differentiating the activity curves as we did in paper I, the activity curve for each case was fit to a cubic function and the thermodynamic factor was determined analytically. The diffusivity calculated following this procedure is shown in Fig. 8, For comparison, the diffusivity calculated from the solvent concentration profile reported in Sec. IVA is also shown as closed symbols. In general, the diffusivity calculated from the two 


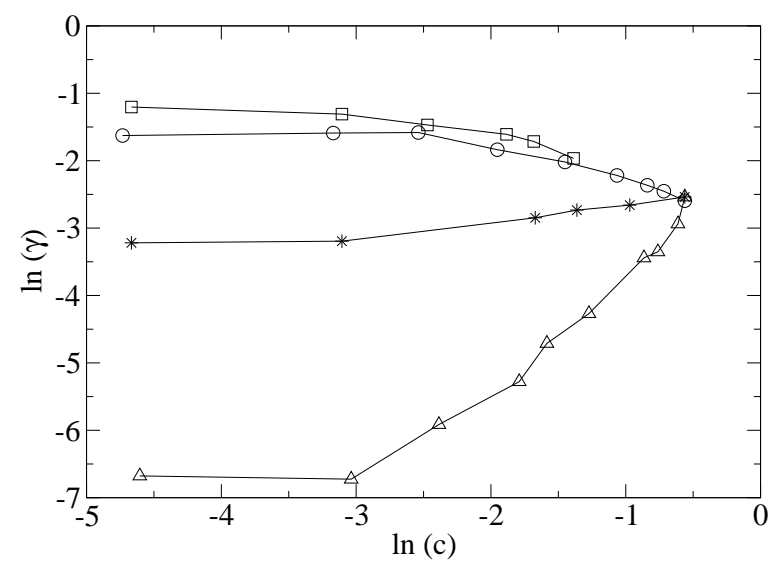

FIG. 7: Activity coefficient of solvent as a function of solvent concentration. Symbols are for $\left(\epsilon_{p p}\right.$, $\left.\epsilon_{s p}\right)=(\epsilon, \epsilon)$ (circles), $(1.33 \epsilon, \sqrt{1.33} \epsilon)$ (squares), $(2.0 \epsilon, 2.0 \epsilon)$ (triangles), and $(1.33 \epsilon, 1.33 \epsilon)$ (stars)

different approaches show good agreement. The diffusivity calculated using the Darken equation shows the expected behavior discussed above, the diffusivity is constant for $\epsilon_{p p}=\epsilon$ and increases exponentially for $\epsilon_{p p}=\epsilon_{s p}=2.0 \epsilon$. However, there is more scatter due to the uncertainty in the thermodynamic factor.

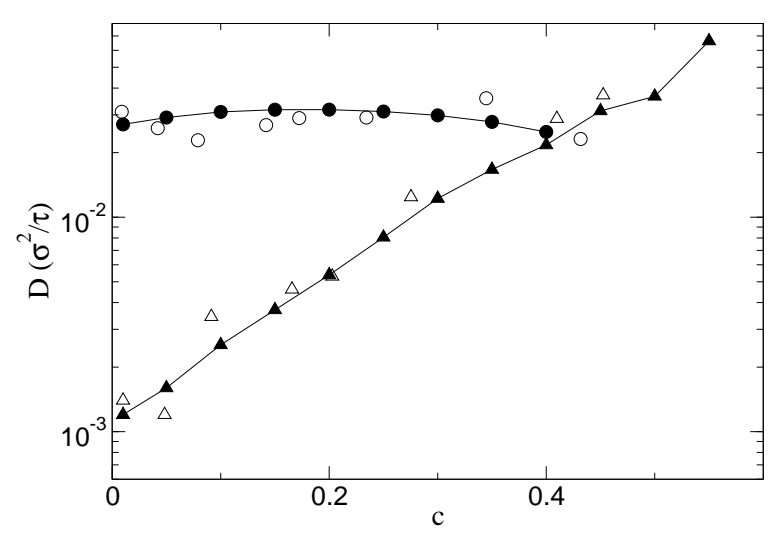

FIG. 8: Diffusivity $D(c)$ as a function of solvent concentration and open symbols are from Darken equation Eq. 4 and closed symbols are from the concentration profile using Eq. 3. Circles and triangles are for $\epsilon_{p p}=\epsilon_{s p}=\epsilon$, and $2.0 \epsilon$, respectively.

In the interdiffusion study the behavior of $D(c)$ is related to the shape of the solvent concentration profile. Similarly, in the present approach the behavior of $D(c)$ can be directly related to the functional form of the activity coefficient. In general, for a constant diffusivity 
the activity coefficient decreases with concentration, and increases with concentration for a diffusivity that increases with concentration.

\section{SUMMARY}

The effect of polymer-polymer and solvent-polymer interactions on the behavior of the interdiffusion of a solvent in to an entangled polymer matrix have been studied using large scale molecular dynamics and grand canonical Monte Carlo simulation techniques. By varying the polymer-polymer interaction the state of the polymer is changed from melt to glassy. Correspondingly the solvent density profile changed from error function like to a sharp front, characteristic of Case II type transport, when Berthelot's rule is applied for the solventpolymer interaction. The weight gain by the polymer matrix increased as $t^{1 / 2}$ in agreement with Fickian diffusion, even for the case of a glassy polymer. This suggests that the precursor of the front is Fickian in agreement with recent experimental observations that characterize Case II diffusion by a sharp concentration front with Fickian type precursor ${ }^{8,15,18,19}$ The front, however, does not move in the time scale of our simulation. From simulation of equilibrated solvent-polymer solution it was found that the glassy system with Berthelot's rule applied for the cross term is immiscible except in the dilute limit suggesting that the front may not move in to the polymer matrix. Increasing the solvent-polymer interaction enhanced the solubility of the system without changing the nature of the diffusion process.

The solvent concentration profiles have been fitted using the one-dimensional Fick's model of the diffusion process. The diffusivity, $D(c)$, shows strong dependence on the state of the polymer. Far above the glass transition $D(c)$ is approximately constant and then becomes concentration dependent as the polymer becomes glassy. The shape of the concentration profile and the behavior of $D(c)$ is found to be directly related. The diffusivity is constant when the solvent concentration profile is concave, shows exponential dependence on solvent concentration when the solvent concentration profile is convex.

The diffusivity as a function of solvent concentration was also determined using the Darken equation for simulations of equilibrated solvent-polymer solution. The diffusivity calculated using this approach is in good agreement with the diffusivity calculated from the solvent concentration profile.

The advantage of the Darken approach for determining $D(c)$ is that it requires much 
smaller system sizes than for the direct simulation of the interdiffusion process. However, each solvent concentration c has to be determined separately and the simulation time required to determine the $D_{c}(c)$ and the fugacity $f$ are quite long compared to the interdiffusion studies. This is because the scaled concentration profiles superimpose even after relatively short times.

\section{ACKNOWLEDGMENTS}

Sandia is a multiprogram laboratory operated by Sandia Corporation, a Lockheed Martin company, for the United States Department of Energy's National Nuclear Security Administration under Contract No. DE-AC04-94AL85000.

1 F. Müller-Plathe, Chem. Phys. Lett. 252, 419 (1996).

2 S. G. Charati and S. A. Stern, Macromolecules 31, 5529 (1998).

3 N. F. A. van der Vegt, Macromolecules 33, 3153 (2000).

4 X. Yi and J. Pellegrino, J. Poly. Sci.:B: Poly. Phys. 40, 980 (2002).

5 S. Y. Lim, T. T. Tsotsis, and M. Sahimi, J. Chem. Phys. 119, 496 (2003).

6 T. P. Gall, R. C. Lasky, and E. J. Kremer, Polymer 31, 1491 (1989).

7 T. R. Cuthbert and N. J. Wagner, Macromolecules 32, 5017 (1999).

8 M. M. Hassan and C. J. Durning, J. Polym. Sci. 37, 3159 (1999).

9 K. S. Kwan, C. N. Subramaniam, and T. C. Ward, Polymer 44, 3061 (2003).

10 N. L. Thomas and A. H. Windle, Polymer 19, 255 (1978).

11 N. L. Thomas and A. H. Windle, Polymer: 21, 613 (1980); 22, 627 (1981); 23, 529 (1982);

12 J. S. Vrentas, J. L. Duda, and W. J. Huang, Macromolecules 19, 1718 (1986).

13 A. G. Webb and L. D. Hall, Polymer 32, 2926 (1991).

14 I. Hopkinson, R. A. Jones, S. Black, D. M. Lane, and P. J. McDonald, Carbohydrate Polymers 34, 39 (1997).

15 M. Sanopoulous and F. Stamatialis, Polymer 42, 1429 (2001).

16 M. L. Greenfield and D. N. Theodorou, Macrol. 34, 8541 (2001).

17 C.-Y. Hui and K.-C. Wu, J. Appl. Phys. 61, 5129 (1987). 
18 D. F. Stamatialis, M. Sanopoulous, and J. H. Petropoulos, Macromolecules 35, 1021 (2002).

19 C. J. Durning, M. M. Hassan, H. M. Tong, and K. W. Lee, Macromolecules 28, 4234 (1995).

20 J. Crank, The Mathematics of Diffusion (Oxford University Press, Oxford, 1975).

21 J. Sonnenburg, J. Gao, and J. H. Weiner, Macromolecules 23, 4653 (1990).

22 H. Takeuchi, R.-J. Roe, and J. E. Mark, J. Chem. Phys. 93, 9042 (1990).

23 P. V. K. Pant and R. H. Boyd, Macromolecules 25, 494 (1992).

24 F. Müller-Plathe, S. C. Rogers, and W. F. van Gunsteren, Macromolecules 25, 6722 (1992).

25 R. M. Sok, H. J. C. Berendsen, and W. F. van Gunsteren, J. Chem. Phys. 96, 4699 (1992).

26 Y. Tamai, H. Tanaka, and K. Nakanishi, Macromolecules 27, 4498 (1994).

27 R. H. Gee and R. H. Boyd, Polymer 36, 1435 (1995).

28 D. Hofman, J. Ulbrich, L. Fritz, and D. Paul, Polymer 37, 4773 (1996).

29 T. Li, D. O. Kildsig, and K. Park, J. Controlled Release 48, 57 (1997).

30 F. Müller-Plathe, J. Chem. Phys. 108, 8252 (1998).

31 O. Hahn, D. A. Mooney, F. Müller-Plathe, and K. Kremer, J. Chem. Phys. 111, 6061 (1999).

32 G. E. Karlsson, T. S. Johansson, U. W. Gedde, and M. S. Hedenqvist, Macromolecules 35, 7453 (2002).

33 M. Tsige and G. S. Grest, J. Chem. Phys. 120, 2989 (2004).

34 A. R. C. Baljon and M. O. Robbins, Macromolecules 34, 4200 (2001).

35 G. S. Grest and K. Kremer, Phys. Rev. A 33, 3628 (1986).

36 K. Kremer and G. S. Grest, J. Chem. Phys. 92, 5057 (1990).

37 S. J. Plimpton, J. Comput. Phys. 117, 1 (1995).

38 G. S. Heffelfinger and D. M. Ford, Mol. Phys. 94, 659 (1998).

39 A. P. Thompson and G. S. Heffelfinger, J. Chem. Phys. 110, 10693 (1999). 